\title{
EL CONCEPTO DE AMBIENTE EN LOS LIBROS DE TEXTO DE CIENCIAS NATURALES
}

\section{THE ENVIRONMENTAL CONCEPT IN TEXTBOOKS OF SCIENCE}

\section{Sismay García Bermúdez ${ }^{1}$}

\section{Resumen}

En este artículo se presentan los resultados de una investigación que tiene como objetivo el análisis de contenido de los libros de texto de los grados 6 y 8 de educación básica secundaria en Colombia, buscando la forma en la que se presenta el concepto de ambiente para los estudiantes y docentes. La investigación está enmarcada en la línea de la investigación documental cualitativa e interpretativa. En el artículo se tratan dos categorías de las planteadas en la totalidad del trabajo: la forma en la que presenta el concepto si es explícita 0 implícita y si se plantea desde una visión físico natural o sistémica.

\section{Summary}

This article presents the results of research that aims at analyzing the content of textbooks in grades 6 and 8 of basic secondary education in Colombia, looking for ways in which we present the concept of environment for students and teachers. The research is framed within the research line qualitative and interpretive documentary. The article addresses two categories of those raised in the entire work: the way in which it presents the concept if it is express or implied and whether it arises from a vision natural physical or systemic.

Palabras Clave: concepto de ambiente, libros de texto, enseñanza de las ciencias.

Keywords: concept of environment, textbooks, science education.

\section{Introducción}

Desde los marcos legales que se han venido planteando, la inclusión de la dimensión ambiental en el sector educativo, parte del reconocimiento de la problemática ambiental local y de la formulación de proyectos integrales e interdisciplinarios, que desde la escuela permitan lecturas contextuales para la comprensión de una problemática y para la ejecución de acciones orientadas a la

\footnotetext{
${ }^{1}$ Magister en Didáctica de las Ciencias Universidad Internacional de Andalucía - Universidad de Huelva Grupo de Investigación INNOVACIENCIA - UdeA sismagarcia@hotmail.com
} 
Memorias del VII Encuentro Nacional de Experiencias en la Enseñanza de la Biología y la Educación Ambiental y II Congreso Nacional de Investigación en la Enseñanza de la Biología

búsqueda de soluciones compartidas y de posible aplicación y proyección, no sólo para los actores de la escuela, sino para todos aquellos que están inmersos en las mismas.

A pesar de esto se observa que estas problemáticas se siguen presentando en todos los sectores, esto tal vez ha ocurrido por el hecho de que los problemas ambientales han sido comúnmente evidenciados por medio de desequilibrios naturales que se presentan a la opinión pública como catástrofes. De esta manera se ha delegado la responsabilidad, en cuanto a gestión del ambiente se refiere, a las personas que de una $u$ otra forma tienen que ver solamente con el manejo de fenómenos naturales; cabe entonces preguntarse si la forma de vincular el tema ambiental en el ámbito escolar está produciendo efectos positivos en la sociedad; por ello hay que analizar de forma más profunda lo que se está enseñando en las escuelas. Los anteriores supuestos llevan a plantear que el trabajo que se debe hacer obliga a apuntar hacia el análisis de los docentes y por ende de los materiales con los que éste trabaja, estando incluidos en éstos los libros de texto, ya que son uno de los elementos más utilizados por los profesores para presentar el conocimiento a sus estudiantes. Desde esta perspectiva la investigación tiene como objetivo central Analizar Cuál es la perspectiva de ambiente planteada en los libros de texto de ciencias naturales utilizados en el ciclo de enseñanza básica secundaria en Medellín y su relación con las políticas curriculares planteadas por el Ministerio de Educación Nacional.

\section{El concepto de ambiente:}

Desde el currículo hay un compromiso para la inclusión de la temática ambiental en la formación de los estudiantes, pero, es necesario recalcar que según la Política Nacional de Educación Ambiental (SINA 2002) las estrategias utilizadas para tal fin están concentradas en aspectos puramente ecológicos dejando de lado lo cultural y social, que hacen parte integral de la problemática ambiental, esto dificulta una visión sistémica del ambiente en los procesos formativos. En este sentido y con el fin de aclarar la concepción actual del ambiente, elabora los siguientes planteamientos:

- El Ambiente, como un sistema dinámico definido por las interacciones físicas, biológicas, sociales y culturales, percibidas o no, entre los seres humanos y los demás seres vivientes y todos los elementos del medio en el cual se desenvuelven, bien que estos elementos sean de carácter natural o sean transformados o creados por el hombre.

- El sistema ambiental, entendido como un conjunto de relaciones en el que la cultura actúa como estrategia adaptativa entre el sistema natural y el sistema social 
Bio-grafía Escritos sobre la Biología y su Enseñanza.

Edición Extra-Ordinaria. ISSN 2027-1034 P. p 141 - 148

Memorias del VII Encuentro Nacional de Experiencias en la Enseñanza de la Biología y la Educación Ambiental y II Congreso Nacional de Investigación en la Enseñanza de la Biología

La problemática ambiental, concebida como un problema social que refleja un tipo de organización particular de la sociedad y una relación específica de esta organización con su entorno natural.

La educación ambiental, considerada como el proceso que le permite al individuo comprender las relaciones de interdependencia con su entorno, a partir del conocimiento reflexivo y crítico de su realidad biofísica, social, económica y cultural para que, a partir de la apropiación de la realidad concreta, se puedan generar en él y en su comunidad actitudes de valoración y respeto por el ambiente.

La integración de cada uno de estos términos o conceptos, hace parte de lo que es la visión sistémica de ambiente, la clasificación anterior es un desglose del concepto que permite visualizar más profundamente lo que se entiende por ambiente y todos los aspectos que lo componen.

Por otro lado, para entender el concepto de ambiente desde una visión sistémica, es necesario saber la definición de sistema, aunque es un concepto complejo, se puede entender así:

“Un sistema está constituido por elementos en interacción, formando un todo de complejidad creciente. Su importancia estriba en las interacciones que los elementos crean entre sí, pues el conjunto de estas interacciones constituye la riqueza del sistema. Los elementos integrados uno dentro de otro son unidades". Aguaded (2008) ${ }^{2}$.

Teniendo en cuenta lo expuesto hasta aquí, se debe comprender que el concepto de ambiente no puede reducirse estrictamente a la conservación de la naturaleza, a la problemática de la contaminación por basuras o a la deforestación, es más amplio y se deriva de la complejidad de los problemas y potencialidades ambientales y del impacto de los mismos no sólo en los sistemas naturales, sino también en los sistemas sociales.

\section{Los libros de texto en el aula de clase}

En la actualidad existen múltiples investigaciones sobre los libros de texto, en ellas se tienen diferentes apreciaciones sobre su uso, en algunos se le da mayor responsabilidad al docente pues es quien determina los materiales a utilizar y el contenido que se entrega en cada clase, por otro lado se responsabiliza a la legislación de cada país, puesto que los libros deben estar de acuerdo con los contenidos determinados por el gobierno.

\footnotetext{
${ }^{2}$ Documentos inéditos, Curso de Educación ambiental, Máster en investigación en enseñanza de las ciencias experimentales, sociales y matemáticas, Universidad De Andalucía, Universidad de Huelva, 2008.
} 
Memorias del VII Encuentro Nacional de Experiencias en la Enseñanza de la Biología y la Educación Ambiental y II Congreso Nacional de Investigación en la Enseñanza de la Biología

Así, Solarte (2006) afirma que los libros de texto son registros de conocimiento de la realidad, estos son considerados como recursos didácticos en la educación, ya que proponen un camino, tienen un enfoque y una perspectiva que marcan el proceso de construcción del conocimiento; además tienen un propósito para la enseñanza que no solo es el de enseñar, sino el de contribuir en la formación para el aprendizaje; esto se logra a través del diseño de actividades al presentar los conceptos los cuales deben influir en el proceso de transformación del pensamiento. De igual modo, se puede decir que el libro de texto tiene un protagonismo bastante alto en el proceso de enseñanza y aprendizaje que se da en el aula y fuera de ella, puesto que la mayoría de los estudiantes reciben la principal formación a través de ellos, la gran cantidad de contenidos que se ven en el aula están determinados por lo que dice el libro de texto ya sea porque se lee directamente de él o porque el docente lo tiene como material de consulta para preparar sus clases. Esto no significa que sea el único material utilizado pero sí uno de los más importantes y más utilizado.

De acuerdo con esto, la Comisión de Educación Ecológica de Madrid (2006), ecologistas en acción, en su estudio el currículo antiecológico de los libros de texto, afirman que: "aunque los libros de texto son solo una fuente entre otras de formación de categorías mentales, son sin embargo representativos de lo que la sociedad considera un conocimiento más "serio" y "objetivo". Para muchas personas lo que dicen los libros de texto son verdades tan incuestionables como que el sol sale por el este. Esto es realmente importante porque en muchas ocasiones el docente (sobre todo aquel que no se ciñe al contenido del libro de texto) hace una afirmación sobre un concepto determinado y si éste se encuentra definido de forma diferente en el texto, los estudiantes afirman que el docente está equivocado porque en el texto dice otra cosa. Por lo tanto los alumnos confían plenamente en lo que sus libros dicen, en definitiva son los textos quienes tienen la última palabra

En este mismo sentido, Pozo y Gómez Crespo (1998) dicen que: "aunque evidentemente no son las únicas causas en la generación de concepciones alternativas durante la etapa de escolarización influyen tanto la experiencia cotidiana como el tratamiento de los conceptos en los libros de texto" además en la etapa de educación primaria, el proceso de enseñanza - aprendizaje tiene un apoyo fundamental y en ocasiones exclusivo, en los libros de texto, puesto que para los docentes la utilización de los mismos les permite llevar al aula más 0 menos transformados, el saber que producen los científicos.

Por consiguiente conviene tener cuidado con esto, porque un concepto planteado erróneamente en un texto puede formar una concepción errada en el estudiante y como se dijo anteriormente, puede ser difícil de cambiar por la "fe" que tienen los alumnos en el conocimiento planteado por los libros. 
Bio-grafía Escritos sobre la Biología y su Enseñanza.

Edición Extra-Ordinaria. ISSN 2027-1034 P. p 141 - 148

Memorias del VII Encuentro Nacional de Experiencias en la Enseñanza de la Biología y la Educación Ambiental y II Congreso Nacional de Investigación en la Enseñanza de la Biología

\section{Metodología}

Con el fin de alcanzar los objetivos propuestos, la metodología que se implementó durante el desarrollo del proyecto, se enmarca dentro de la investigación documental y el análisis de contenido, cualitativa e interpretativa, ésta se caracteriza por la revisión y análisis de diferentes fuentes bibliográficas para emitir un juicio final o unas conclusiones sobre el tema que se investiga.

La población consta de 30 libros de ciencias naturales de educación básica secundaria de los grados sexto y octavo. Se seleccionaron cuatro editoriales, tres de ellas por ser las más utilizadas en los colegios e institutos educativos de la ciudad de Medellín, en su orden de importancia son: Santillana, Norma S.A, Voluntad S.A y por último McGraw Hill, que no es muy usada pero sirve como material de consulta para los estudiantes y docentes en las bibliotecas escolares y públicas. Posteriormente se realiza la revisión del concepto de ambiente en cada uno de los textos, teniendo en cuenta unos criterios; que a su vez, se convierten en las categorías de análisis: para este artículo solo se tendrán en cuenta dos categorías, la forma en la que aparece (si es implícito o explícito) y la concepción que se tiene del ambiente, si es tratado desde una visón sistémica o por el contrario desde una visión físico natural.

Para el análisis de la información se hace triangulación entre editoriales, entre libros de la misma editorial y entre grados, analizando contenidos, variables y generalidades y las categorías definidas en el instrumento de recolección de datos, se elabora una tabla de compilación de los datos encontrados, la tabla de categorías y sus definiciones.

\section{Resultados y discusiones}

La categoría inicial define si se encuentra de forma explícita o implícita, teniendo en cuenta que la forma en la que aparece en el libro de texto, facilita la búsqueda del mismo a la hora de ser consultado por cualquier estudiante o docente.

En 5 textos, es decir el $17 \%$ de la muestra, se encuentra el ambiente definido explícitamente; en 24 libros que representan el $80 \%$ de los 30 textos analizados, aparece el ambiente como un concepto definido implícitamente, puesto que es necesario analizar el contexto en el que se encuentra la palabra para poder definir su significado, se hacen más bien comparaciones o se presenta como sinónimo de ecosistema o de un factor que hace parte de él, a pesar de que en los títulos de las unidades aparece como un factor que se supone será tratado directamente a lo largo de las mismas. No hay definición del concepto analizado, todos los apartados en los que aparece la palabra ambiente se encuentran relacionados con el espacio físico en el que viven los seres vivos y no vivos. 
Memorias del VII Encuentro Nacional de Experiencias en la Enseñanza de la Biología y la Educación Ambiental y II Congreso Nacional de Investigación en la Enseñanza de la Biología

En general, en los libros de octavo grado, se encuentra en mayor porcentaje $(43 \%)$ la definición implícita, según la comparación entre grados, el texto Cosmos 6. Ciencias interactivas 8 y Conciencia 8 presentan el concepto implícito y estos mismos textos en los grados 8 y 6 respectivamente los plantean de forma explícita; esto indica que no se sigue una secuencia lógica ni se plantea un encadenamiento en los contenidos que se trabajan según las temáticas planteadas en cada uno. El resto de los libros se encuentran de forma similar en ambos grados, excepto Biociencias 6 que no presenta definición del término y en el grado 8 sí está definido.

\section{Concepción del ambiente: sistémica o físico - natural}

Esta categoría tiene que ver con la forma en la que se concibe el ambiente dentro del libro de texto. En el marco referencial se determina cada uno de los componentes de forma amplia. La visión sistémica tiene que ver con la forma holística de definir el ambiente, es decir, se tienen en cuenta los cuatro componentes: físico, natural, social y cultural. En la visión físico - natural solo se tiene en cuenta estos dos aspectos, dejando de lado lo social y cultural.

Según los resultados obtenidos en 29 libros, el 97\% de los 30 textos analizados se define el ambiente desde una visión físico - natural y en 1 libro, el $3 \%$ del total de textos, se plantea según la visión sistémica. En los textos que aparece según la visión físico - natural, en general se plantea como lugar o espacio en el que se dan diversas relaciones entre los seres vivos y los no vivos. Haciendo una clasificación de las definiciones que se encuentran en los libros de texto analizados y ubicándolos en cada una de las categorías planteadas en el Esquema 1, se puede encontrar que en la mayoría de las definiciones se utilizan términos pertenecientes a la concepción naturalista y tecnológica y solo en una de las definiciones se utiliza un término relacionado con la concepción ética, en seis definiciones hay términos relacionados con la concepción práctica y en tres de ellos hay expresiones relacionados con la concepción política y social.

Teniendo en cuenta lo expuesto anteriormente, al caracterizar y analizar el concepto de ambiente presente en los libros de texto de ciencias naturales de los grados 6 y 8 de educación básica secundaria, utilizados en la ciudad de Medellín (Colombia) se encuentra que el $97 \%$ de los textos trata el tema ambiental y solo el $3 \%$ no presentan una temática enfocada hacia el ambiente. En aquellos libros en los que se trata el tema ya sea de forma implícita o explícita se deja sin explicar los componentes básicos del concepto de ambiente visto desde una perspectiva holística que integre la cultura, la política, el aspecto bioquímico, biológico y social del mismo.

La falta de estructura temática en los libros de texto puede generar dificultades desde varios puntos de vista tanto para los docentes como para los estudiantes que los utilizan, por ejemplo para los docentes deja abierta la posibilidad de 
Memorias del VII Encuentro Nacional de Experiencias en la Enseñanza de la Biología y la Educación Ambiental y II Congreso Nacional de Investigación en la Enseñanza de la Biología

interpretar el significado del concepto de ambiente a su amaño, sin tener en cuenta los aspectos que conforman el ambiente como sistema, además desde la misma definición y la presentación implícita del concepto hay ambigüedades; esto puede causar problemas en la forma en la que se enseña el tema, puesto que en la mayoría de los casos el libro de texto es la herramienta de consulta y preparación de la clase más utilizada por el profesor.

Para el estudiante es en igual medida problemático, porque no hay claridad en la definición de los conceptos, además de ser muy corta la presentación del tema sobre todo cuando se incluye en definiciones de otros conceptos afines al tema. Para ambos actores del proceso de enseñanza aprendizaje puede quedar la concepción de que el ambiente es un espacio físico y nada más.

Con base en estas ideas, se hace necesario que las editoriales o más bien los autores de los textos, diseñen los libros de acuerdo a ciertos parámetros entre los que se debe incluir una actualización del conocimiento sobre la concepción de ambiente, puesto que es un tema que desde la legislación de Colombia se considera de enseñanza obligatoria en todos los grados de educación básica y media.

\section{Consideraciones generales}

Para los estudiantes el ambiente no es parte de su vida diaria, lo excluyen puesto que se piensa que no desperdiciar el agua y reciclar es una buena forma de contribuir con el cuidado del ambiente. En su mente aún no está que el cuidado de las relaciones personales, su autocuidado, el respeto por los demás y por su propia vida, además el docente en ocasiones no se plantea la pregunta de ¿qué es para el estudiante el ambiente? ¿Cómo se ve él en el ambiente, cuál es su papel dentro de éste? Simplemente se lo presenta como algo aislado de su vida, así como se encuentra en los libros de texto.

En la enseñanza de los conceptos en la clase de ciencias se debe tener en cuenta las ideas previas de los estudiantes para identificar lo que cada uno sabe y así enseñar de acuerdo a esto. Pero por lo general al estudiante se le presenta el concepto sin antes indagar por lo que no se consigue una interiorización y una posible evolución conceptual que luego proporcione un cambio en la visión que tiene el alumno sobre el concepto (en este caso el ambiente) enfrentado a su realidad para modificarla.

Es entonces un problema que tiende a continuar si los docentes que son los que utilizan los libros de texto no se interrogan y utilizan otros medios y métodos de enseñanza o si no solicitan que las personas que escriben los libros de texto se documentan y cambian la forma de presentar los conceptos en sus libros. Aunque es más necesario que los docentes no se peguen directamente a estos textos y 
Bio-grafía Escritos sobre la Biología y su Enseñanza.

Edición Extra-Ordinaria. ISSN 2027-1034 P. p 141 - 148

Memorias del VII Encuentro Nacional de Experiencias en la Enseñanza de la Biología y la Educación Ambiental y II Congreso Nacional de Investigación en la Enseñanza de la Biología

elaboren sus propias clases de acuerdo al conocimiento que se tienen de los conceptos que se deben enseñar en clase.

\section{Referencias bibliográficas}

AGUADED, J. S. (2006) Selección de Textos Escolares Como parte de la Gestión Curricular. España: Curso de educación ambiental Máster Universidad Internacional De Andalucía.

COMISION DE EDUCACION ECOLOGICA, ECOLOGISTAS EN ACCION (2006) Madrid. El Estudio del Curriculum Oculto Antiecológico de los Libros de Texto. unw.ecologistasenaccion.org/curriculumoculto

FONTAINE, L. y EYSAGUIRRE, B. (1997) Por qué es Importante el texto Escolar. Chile: Estudios públicos 68 (primavera 1997)

GUEMES, R. (1994) Algunas Investigaciones en Torno al Uso de los Libros de Texto. Departamento de Didáctica e Investigación Educativa y del comportamiento. Universidad de la Laguna. Comunicación y Pedagogía, Nuevas Tecnologías y Recursos Didácticos. N¹57. 76-83

REPUBLICA DE COLOMBIA (1994) Ley General de Educación o Ley 115 febrero 8 de 1994. Bogotá: Ministerio de Educación Nacional.

REPÚBLICA DE COLOMBIA (1994) Decreto 1743 del 3 de agosto de 1994. Bogotá: Ministerio de Educación Nacional y Ministerio del Medio Ambiente.

SISTEMA NACIONAL AMBIENTAL DE COLOMBIA (2002) Política Nacional de Educación Ambiental. Bogotá: Ministerio del Medio Ambiente y Ministerio de Educación Nacional.

SOLBES, J. y VILCHES, A. (2004) Papel de las relaciones entre ciencia, tecnología, sociedad y ambiente en la formación ciudadana. Enseñanza de las Ciencias, 22 (3), 337-348 